\title{
A Method of Abstract Service Aggregation for Service Composition in Service oriented Manufacturing System
}

\author{
Hua Guo ${ }^{1, a^{*}}$, Min Shu ${ }^{2, b}$ \\ ${ }^{1}$ College of Information Science and Engineering, Shandong Agricultural University, Taian, China \\ ${ }^{2}$ College of Mechanical and Electronic Engineering, Tanshan Polytechnic, Taian, China \\ aguoh@sdau.edu.cn, b shuchang198010@163.com
}

Keywords: abstract service aggregation, composable correlation, service composition, service oriented manufacturing

Abstract. Service composition, which can realize the added value of service, is the core to implement service oriented manufacturing system. The paper mainly studies the method of abstract service aggregation based on composable correlation drove by the user's service request. First, the several related definitions are illustrated. Then service aggregation algorithm is given by employing partial ordering relationship of abstract service discovered base on the composable correlation among services.

\section{Introduction}

Service oriented manufacturing is a kind of new business model, production organization manner and operation pattern. When service requester submits complex tasks that single service in service center cannot meet their requirements, and then it needs to combine several service to form more powerful composite service to achieve the purpose of added-value of service, i.e. service composition [1,2]. During employ service composition to complete a task, a series of services are needed to be assembled according to certain flow and rule to construct a service composition path to serve the task.

At present, there exist many researches on the service composition, which can be considered for two kinds. One is to directly generate service composition plan for task base on concrete services $[3,4]$. The advantage of this kind of method is that it can immediately carry on service composition and does not need predefined service composition template, which can realize the automation of service composition. But in the practical network, there exist massive concrete services, so this method may limit to the efficiency of service composition. Another kind of method is to match the concrete services in the system for the existing service composition template that constructed by abstract services [5]. In order to realize this method, the construction of service composition template needs human intervention, i.e. aggregation of abstract service, generation and instantiation of service composition path, and so on.

Composable correlation means that there is some similarity between input parameters and output parameters of two services [6]. Therefore, this paper considers composable correlation among services, and presents abstract service aggregation algorithm by the interface matching according to the function description among services which can be employed to construct service composition template.

\section{The Related Definitions}

Definition 1 (Service). It is the functional description (e.g. some performance parameter of service executing), which can be describes as follows:

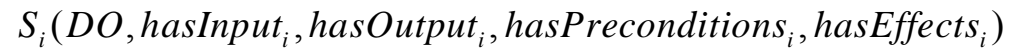

For simplicity, the above expression is denoted by $S_{i}=\left(D O, I_{i}, O_{i}, P_{i}, E_{i}\right)$, where $D O$ is the domain ontology model that $S_{i}$ belongs to. In this paper, it assumed that all the concepts come from the same ontology. $I_{i}=\left\{I_{i_{1}}, I_{i_{2}}, \mathrm{~L}, I_{i_{n}}\right\}$ is the input set and $O_{i}=\left\{O_{i_{1}}, O_{i_{2}}, \mathrm{~L}, O_{i_{m}}\right\}$ is the output set of $S_{i}$, and $n$ and 
$m$ are the number of input parameters and output parameters, respectively, $I_{i_{k}}$ and $O_{i_{k}}$ can be expressed as triples $I_{i_{k}}=$ <concepts, properties, values $>$ and $O_{i_{k}}=$ <concepts, properties, values $>$, $P_{i}=\left\{P_{i_{1}}, P_{i_{2}}, \mathrm{~L}, P_{i_{l}}\right\}$ is the preconditions of $S_{i}$, and $E_{i}=\left\{E_{i_{1}}, E_{i_{2}}, \mathrm{~L}, E_{i_{h}}\right\}$ is the execution effects of $S_{i}$.

Definition 2 (Composable correlation) [6]. In services composition, let $S_{j}$ be the successor of $S_{i}$, and composable correlation is defined as whether there exists data logical correlation between $S_{i}$ and $S_{j}$, i.e., whether there exists a certain similarity in concepts and properties between $S_{i}$ 's output $O_{i}$ and $S_{j}$ 's input $I_{j}$. Composable correlation degree is the degree of their similarity.

Definition3 (The control structure of service composition). It is the execution logic structure, which can be divided to four structures: sequence, parallel, branching and cycle (see Fig.1). The structure of other most service composition is combined by the four structures.

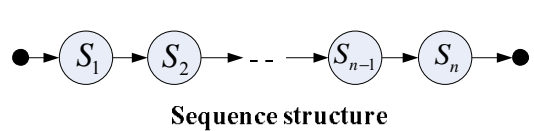

Sequence structure

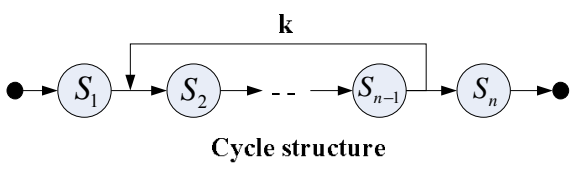

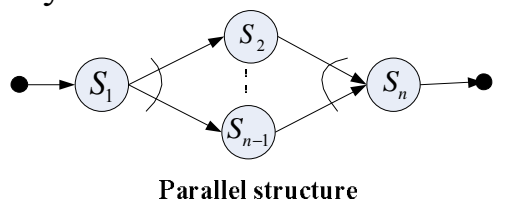

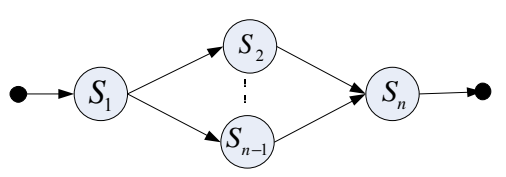

Branching structure

Fig. 1. The basic control structure of service composition

\section{Abstract Service Aggregation Based on Composable Correlation}

Given service request $\mathrm{R}$ and ordered service set $S=\left\{S_{1}, S_{2}, \mathrm{~L}, S_{n}\right\}$. For any service $S_{i}$ and $S_{j}$ with composable correlation in service set $S=\left\{S_{1}, S_{2}, \mathrm{~L}, S_{n}\right\}$, composable correlation between $S_{i}$ and $S_{j}$ is denoted as $S_{i} \rightarrow S_{j}$, where $S_{j}$ is the direct successor service of $S_{i}$ and $S_{i}$ is the direct precursor service of $S_{j}$, i.e. output of $S_{i}$ and input of $S_{j}$ have a certain similarity.

In order to form service composition path, first composable correlation among service set $S$, i.e. all the possible successor service and precursor service for each service are needed to be found. Here, service request is also regarded as a service with input and output function. Then the abstract service aggregation algorithm based on composable correlation is shown in Fig 2. 


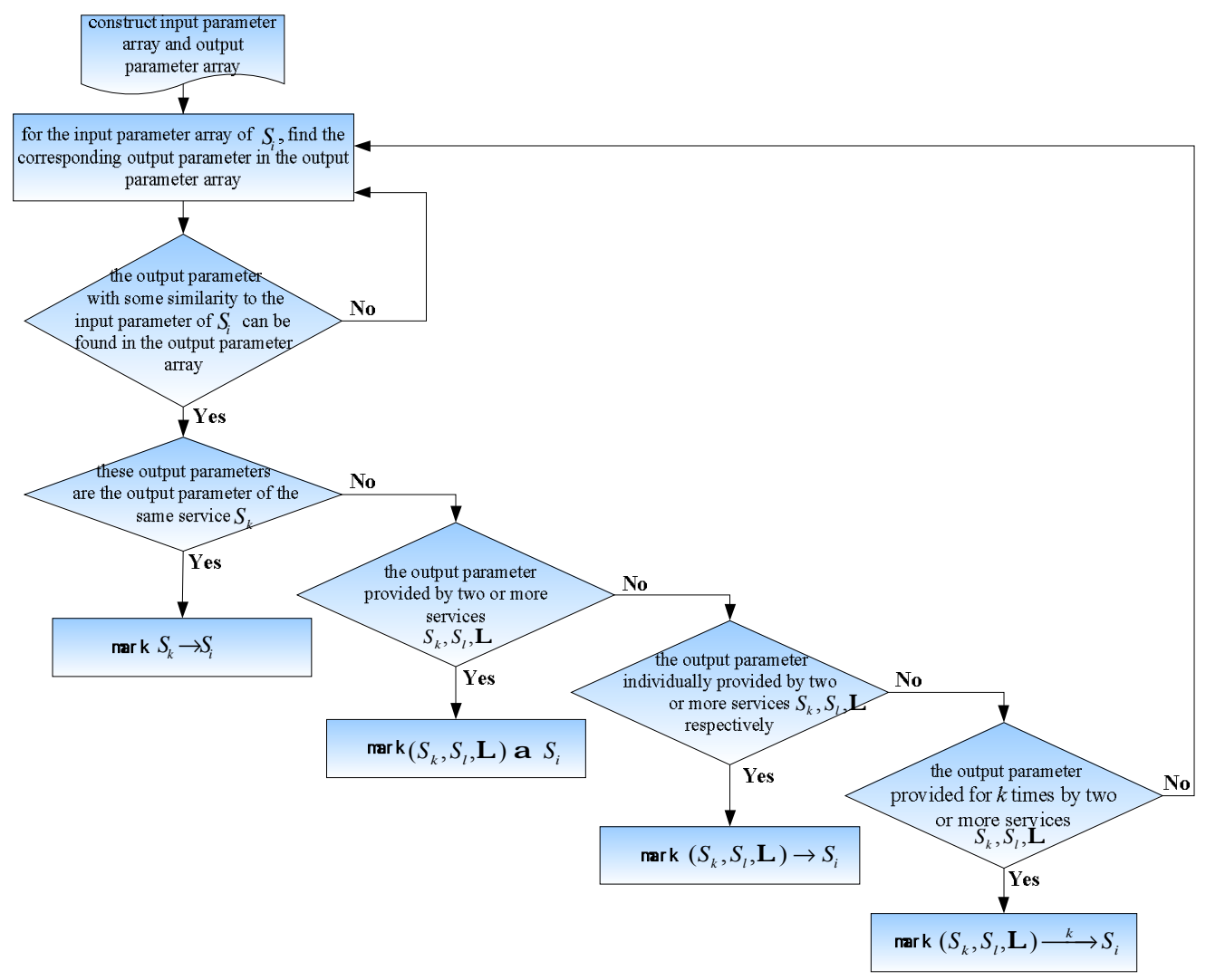

Fig. 2. The abstract service aggregation algorithm based on composable correlation

The main step of abstract service aggregation algorithm is as follows.

Step 1. Construct two arrays, one is the input parameter array and another is the output parameter array.

Step 2. For the input parameter array of each service $S_{i}$, find the corresponding output parameter in the output parameter array.

Step 3. If the output parameter with some similarity to the input parameter of $S_{i}$ can be found in the output parameter array, then check these output parameters whether are the output parameter of the same service.

Step 4. If they are the output parameter of the same service $S_{k}$, then $S_{i}$ and $S_{k}$ is the sequence relationship marked as $S_{k} \rightarrow S_{i}$ (see Fig 3(a)).

Step 5. If they are provided by two or more services $\left(S_{k}, S_{l}, \mathrm{~L}\right)$, then services $S_{k}, S_{l}, \mathrm{~L}$ and service $S_{i}$ are parallel relationship marked as $\left(S_{k}, S_{l}\right.$, L ) a $S_{i}$ (see Fig 3(b)).

Step 6. If they are individually provided by two or more services $\left(S_{k}, S_{l}, \mathrm{~L}\right)$ respectively, then services $S_{k}, S_{l}, \mathrm{~L}$ and service $S_{i}$ are branching relationship marked as $\left(S_{k}, S_{l}, \mathrm{~L}\right) \rightarrow S_{i}$ (see Fig 3(c)).

Step 7. If they are provided for $k$ times by two or more services $\left(S_{k}, S_{l}, \mathrm{~L}\right)$, then services $S_{k}, S_{l}, \mathrm{~L}$ and service $S_{i}$ are cycle relationship marked as $\left(S_{k}, S_{l}\right.$, L ) $\stackrel{k}{\longrightarrow} S_{i}$ (see Fig 3(d)).

Step 8. When all the elements in all input parameter array are searched, the algorithm is over. 


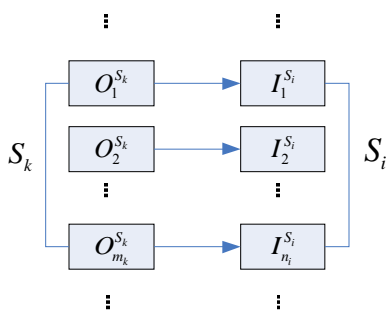

\begin{tabular}{c|c|}
\cline { 2 - 2 }$S_{l}$ & $O_{1}^{S_{l}}$ \\
\hline$O_{2}^{S_{l}}$ \\
\hline$\vdots$ \\
$O_{m_{l}}^{S_{l}}$ \\
\hline
\end{tabular}

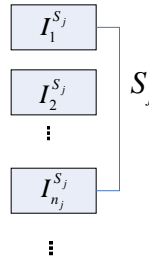

(a)

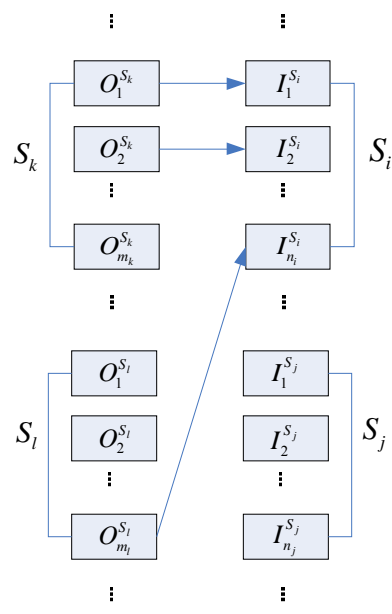

(b)

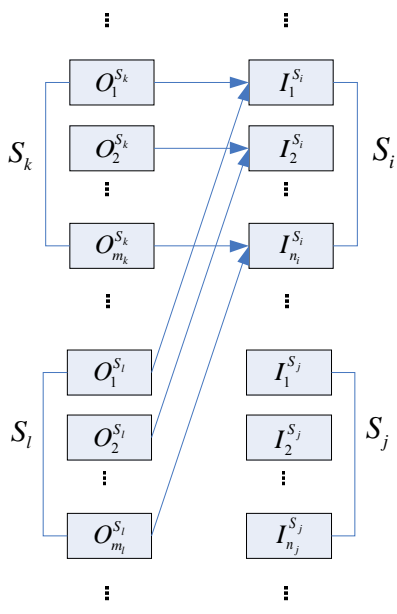

(c)

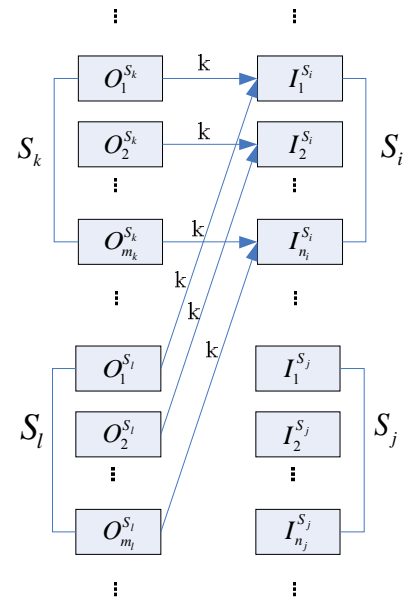

(d)

Fig. 3. The example of abstract service aggregation algorithm

\section{Conclusion}

In this paper, the problem of abstract service aggregation was studies based on composable correlation among services drove by the user's service request in service-oriented manufacturing system. The partial ordering relation of abstract service was discovered base on the composable correlation among services which can realize service aggregation. Meantime, an example was given to illustrate the construction process. In the further, generation and instantiation of service composition path will be given to realize the optimal selection of service composition.

\section{Acknowledgment}

This work is partly supported by Promotive Research Fund for Excellent Young and Middle-aged Scientisits of Shandong Province (NO. BS2014ZZ012), a Project of Shandong Province Higher Educational Science and Technology Program (NO. J14LN36), and the Innovation Foundation of Shandong Agricultural University for Young Scholar.

\section{References}

[1] Y.S. Luo, Kun Yang, Q. Tang, J.M. Zhang, B. Xiong, A multi-criteria network-aware service composition algorithm in wireless environments, Computer Communications, 35(2012)1882-1892.

[2] W. Zhou, J.H. Wen, M. Gao, J.W. Liu, A QoS preference-based algorithm for service composition in service-oriented network, Optik, 124(2013)4439-4444.

[3] R.P. Singh, K.K.Pattanaik, An Approach to Composite QoS Parameter based Web Service Selection, Procedia Computer Science, 19(2013)470-477.

[4] F.Tao, D.M. Zhao, Y.F. Hu, Z.D. Zhou, Correlation-aware resource service composition and optimal selection in manufacturing grid, European Journal of Operational Research, 201(2009)129-143.

[5] B. Benatallah, Q.Z. Sheng, M. Dumas, The self-serv environment for web services composition, IEEE Internet Computing, vol(2003)40-48.

[6] H. Guo, F. Tao, L. Zhang, S.Y. Su, N. Si, Correlation-aware web services composition and QoS computation model in virtual enterprise, International Journal of Advanced Manufacturing Technology, $51(2010) 817-827$. 